\title{
Prevalence and Predictors of Menstruation-related School Absenteeism Among Adolescent Girls in Rural Northern Ghana
}

Maxwell Tii Kumbeni ( $\nabla$ tiimax2@gmail.com )

Ghana Health Service https://orcid.org/0000-0001-5525-4022

Florence Assibi Ziba

University for Development Studies

Joana Apenkwa

Kwame Nkrumah University of Science and Technology

Easmon Otupiri

Kwame Nkrumah University of Science and Technology

\section{Research}

Keywords: Adolescent girls, menstruation, school absenteeism, prevalence, predictors, Ghana

Posted Date: January 18th, 2021

DOI: https://doi.org/10.21203/rs.3.rs-146221/v1

License: (c) (i) This work is licensed under a Creative Commons Attribution 4.0 International License. Read Full License 


\section{Abstract}

Background: Although menstruation is a normal physiological process that begins in girls during adolescence, it has the potential to negatively impact on the self-esteem and education of girls particularly those from low- and middle-income countries. We investigated the prevalence and predictors of menstruation-related school absenteeism among adolescent girls in the Talensi district of rural northern Ghana.

Methods: We conducted a cross-sectional survey among 705 adolescent girls aged 12-19 years who had attained menarche. The sample size was estimated using Epi Info version 6 at 95\% confidence interval and a $5 \%$ margin of error. A two-stage sampling technique was employed to recruit participants. We conducted univariate and multivariate logistic regression models to determine factors associated with menstruation-related school absenteeism which was defined as "being absent from school due to menstruation-related issues during the last menstruation."

Results: The prevalence of menstruation-related school absenteeism was $27.5 \%$. School absenteeism ranged from one to seven days during the menstrual period. Older adolescent girls, (aOR=2.38, 95\% Cl: $1.29-4.40)$, use of cloth as a sanitary material at the last menstruation, (aOR=3.21, 95\% $\mathrm{Cl}: 2.22-4.63)$, and cultural restriction, (aOR=2.54, 95\% Cl: $1.76-3.67)$ were associated with higher odds of menstruation-related school absenteeism. Meanwhile, girls from moderate income parent(s), [aOR=0.57 $95 \% \mathrm{Cl}: 0.34-0.94]$ had lower odds of menstruation-related school absenteeism. Mother's education and privacy in school were only significant at the univariate level.

Conclusions: The prevalence of menstruation-related school absenteeism highlights the need for interventions aimed at improving the availability of sanitary pads for girls, eliminating cultural restrictions associated with menstruation, and also improving parent(s) income level.

\section{Plain English Summary}

Menstruation-related school absenteeism among adolescent girls in low-and-middle income countries become a widespread challenge. Some adolescent girls miss school up to seven days during their menstrual periods, and this has the potential to stamped their academic progress. Challenge such as lack of sanitary pads, inadequate water sanitation and hygiene facilities, cultural restrictions and teasing from boys are faced by girls during menstruation.

We interviewed adolescent girls who had ever menstruated in some selected schools in rural Northern Ghana. Information on their demographic characteristics and those of their parents were assessed. We also gathered information relating to their menstruation and challenges they encounter in school during their menstrual period. About 705 of the girls completed the self-administered questionnaire.

One out of every four adolescent girls absented themselves from school during their last menstrual period. Most of these girls spent one to four days away from school while a few of them spent between 
five to seven away from school. The older adolescent girls, those who used cloth as their menstrual material in their menstruation and those who faced cultural restrictions were mostly the ones who absented themselves from school. The girls whose parent(s) had moderate income were mostly in school during their menstrual period compared to those whose parent(s) had low income.

In effect, menstruation-related school absenteeism is a challenge facing many adolescent girls in rural Northern Ghana. Efforts should be aimed at addressing issues such as lack of sanitary pads, cultural restriction and also improve the economic status of parents in rural areas.

\section{Introduction}

The population of adolescents represent $23 \%$ of the 1.1 billion people living in sub-Saharan Africa, and about a half of them are girls between the ages of 10-19 years [1]. Adolescence is an important milestone which occurs with psychological and biological changes that require prompt sexual and reproductive health (SRH) attention [2]. However, many adolescent girls in low- and middle-income countries (LMICs) face numerous challenges with regards to SRH services [3, 4]. Although there are global efforts to improve educational opportunities for adolescent girls, one of the challenges these girls in LMICs face is managing their menstruation amid other pubertal changes [5-7].

Several studies have documented the impact of menstruation on the education of adolescent girls, with challenges of menstrual hygiene management affecting girls' school attendance. For instance, a recent study in Uganda by Miiro and colleagues [8], suggests that the prevalence of school absenteeism among girls during menstruation was $59 \%$. These girls missed school from one to seven days in a month during their menstrual period. According to Mohammed and others, the prevalence of menstruation-related school absenteeism in Ghana is $40 \%$ [9]. Montgomery and colleagues [10], also reported that school girls stay away from school for up five days in a month during their menstrual period in Ghana. Other studies have reported similar findings in most LMICs $[5,7,11]$.

In the school setting, lack of clean, private, gender specific, water, sanitation and hygiene (WASH) facilities are documented to be associated with menstruation-related school absenteeism among girls $[10,12-14]$. Lack of sanitary pads has also been widely reported to be a major contributory factor to school absenteeism among girls in their menstrual period $[7,10,14,15]$. Other documented factors such as cultural restrictions, age of girls and mother's education are said to play an influence on menstruationrelated school absenteeism $[7,16]$. Self-reported factors such as menstrual pain, fear of staining clothing and fear of being teased at school have also been reported to be associated with school absenteeism [8, $9,17]$.

There are limited studies in Ghana on menstrual issues among school going adolescent girls. More so, these studies have focused on dysmenorrhea, menstrual knowledge and menstrual hygiene management [16-18]. The limited studies that have reported on menstruation-related school absenteeism in Ghana have only assessed sanitary pad with menstrual education as the predictors of school absenteeism [9, 10]. An assessment and understanding of the full range of predictors is an important step towards 
developing specific interventions to curb the rather high burden of menstruation-related school absenteeism. The aim of our study was to investigate the prevalence and predictors of menstruationrelated school absenteeism among adolescent girls in a rural setting of northern Ghana. The findings will be relevant towards improving menstrual health and education of adolescent girls particular in poor and rural settings.

\section{Materials And Methods}

\section{Study design and population}

A cross-sectional study was conducted in junior high schools in the Talensi district of Ghana, from July to September, 2016. A total of 705 school girls aged 12-19 years who had attained at least their first menstruation participated in the study.

\section{Sample size and Data collection}

Epi Info Version 6 was used to calculate the sample size at a prevalence of $50 \%$, using $95 \%$ confidence interval and a $5 \%$ margin of error. The estimated sample was 730 considering a design effect of two and a $10 \%$ non-response rate. A two-stage sampling strategy was employed to select participants. Systematic random sampling was used to select 15 schools at stage one while simple random sampling was used at second stage to select the study participants. The sampling was done with proportionate allocation of size to the study populations in the schools.

\section{Outcome variable}

The outcome variable of interest was menstruation-related school absenteeism. This was defined as "being absent from school due to menstruation-related issues during the last menstruation." Our outcome variable was categorized as "yes" for school absenteeism and "no" for no school absenteeism.

\section{Predictor variables}

The predictor variables were age, religion, mother's education, parent(s) income status, type of sanitary material used during the last menstruation, privacy in school and cultural restrictions. Age was categorized as, 12-15, 16-17, 18-19; religion (Christianity, Islam, Traditional), and mother's education (no formal education, primary education, secondary or higher education). Parent(s) income status was categorized into low, moderate and high, and the type of sanitary material used during the last menstruation (sanitary pad, cloth). Privacy in school was defined as "toilet facility or room with roof and door locks which is accessible to only females" and was categorized as "yes" and "no". Cultural restriction was also defined as any one or more of the following restrictions during menstruation "prevented from going to school, prevented from visiting religious grounds, prevented from playing with colleagues and prevented from eating certain types of foods." "yes" was assigned to restrictions during menstruation and "no" to not being restricted. The variable selection was informed by previous studies $[10,12,14,15]$. 


\section{Data analysis}

Data were analyzed using STATA Version 15 (College Station, TX: Statacorp LLC). Univariate and multivariate analyses were done to assess the relationships between the predictors and our outcome variable. Statistical significance was considered at a $p$-value of $<0.05$.

\section{Results}

\section{Demographic characteristics and predictors of school absenteeism}

Majority (53.3\%) of the girls were aged $16-17$ years, and $92.9 \%$ of them were Christians. Most of the girls' mothers had no formal education (74.8\%), and majority $(73.5 \%)$ of the girls came from low-income status parent(s). About two-thirds (65.5\%) of the girls used sanitary pads during their last menstruation, $65.7 \%$ said they had privacy in school during menstruation and $43.5 \%$ indicated to have faced cultural restrictions during their menstrual periods. The prevalence of school absenteeism by girls due to menstruation was $27.5 \%$. The number of days absent from school in the last menstruation were; one to two days (49.0\%), three to four days (37.1\%) and five to seven days (13.9\%) [Table 1]. 
Table 1

Demographic characteristic and predictors of school absenteeism $(n=705)$

\begin{tabular}{|c|c|}
\hline Variables & Frequency (\%) \\
\hline \multicolumn{2}{|l|}{ Age } \\
\hline $12-15$ & $258(36.6)$ \\
\hline $16-17$ & $376(53.3)$ \\
\hline $18-19$ & $71(10.1)$ \\
\hline \multicolumn{2}{|l|}{ Religion } \\
\hline Christianity & $655(92.9)$ \\
\hline Islamic & $35(5.0)$ \\
\hline Traditional & $15(2.1)$ \\
\hline \multicolumn{2}{|l|}{ Mother's education } \\
\hline No formal education & $527(74.8)$ \\
\hline Primary education & $154(21.8)$ \\
\hline Secondary or higher education & $24(3.4)$ \\
\hline \multicolumn{2}{|l|}{ Parent(s) income status } \\
\hline Low & $518(73.5)$ \\
\hline Moderate & $153(21.7)$ \\
\hline High & $34(4.8)$ \\
\hline \multicolumn{2}{|c|}{ Type of sanitary material used during the last menstruation } \\
\hline Sanitary pad & $464(65.8)$ \\
\hline Cloth & $241(34.2)$ \\
\hline \multicolumn{2}{|l|}{ Privacy in school } \\
\hline No & $242(34.3)$ \\
\hline Yes & $463(65.7)$ \\
\hline \multicolumn{2}{|l|}{ Cultural restrictions } \\
\hline No & 398 (56.5) \\
\hline Yes & 307 (43.5) \\
\hline \multicolumn{2}{|l|}{ School absenteeism } \\
\hline No & $511(72.5)$ \\
\hline
\end{tabular}




\begin{tabular}{|ll|}
\hline Variables & Frequency $(\%)$ \\
\hline Yes & $194(27.5)$ \\
\hline Number of days absent in a month $(n=194)$ & \\
\hline $1-2$ days & $95(49.0)$ \\
\hline $3-4$ days & $72(37.1)$ \\
\hline $5-7$ days & $27(13.9)$ \\
\hline
\end{tabular}

\section{Predictors of school absenteeism}

Girls aged 18-19 years were 2.38 times more likely to have stayed away from school during their menstrual period compared to those aged $12-15$ years, $(\mathrm{aOR}=2.38,95 \% \mathrm{Cl}: 1.29-4.40)$. The girls with parents of moderate-income status had $43 \%$ lower odds of staying away from school during their menstruation when compared with those from low-income status, (aOR $=0.57,95 \% \mathrm{Cl}: 0.34-0.94)$. Additionally, the girls who used cloth as sanitary material in their last menstruation were 3.21 times more likely to have been absent from school during their menstruation compared with those who used sanitary pad, $(\mathrm{aOR}=3.21,95 \% \mathrm{Cl}: 2.22-4.63)$. The girls who encountered cultural restrictions during their menstrual period were significantly more likely to be absent from school when compared with those who did not encounter any cultural restrictions, $(\mathrm{aOR}=2.5495 \% \mathrm{Cl}: 1.76-3.67)$. Mother's education and privacy in school were significant at univariate level but lost significance in the multivariate model (Table 2). 
Table 2

Predictors of school absenteeism $(n=705)$

\begin{tabular}{lll} 
Variables & $\begin{array}{l}\text { Unadjusted or }(95 \% \\
\mathrm{Cl})\end{array}$ & $\begin{array}{l}\text { Adjusted or (95\% } \\
\mathrm{Cl})\end{array}$ \\
\hline Age & 1.00 & 1.00 \\
\hline $12-15$ & $1.52(1.05-2.21)$ & $1.49(0.99-1.23)$ \\
\hline $16-17$ & $2.54(1.44-4.50)$ & $2.38(1.29-4.40)^{*}$ \\
\hline $18-19$ & & \\
\hline Religion & 1.00 & 1.00 \\
\hline Christianity & $1.40(0.68-2.69)$ & $0.99(0.45-2.17)$ \\
\hline Islamic & $1.35(0.45-4.00)$ & $1.40(0.43-4.49)$ \\
\hline Traditional & & 1.00 \\
\hline Mother's education & 1.00 & $0.71(0.44-1.15)$ \\
\hline No formal education & $0.57(0.37-0.89)$ & $0.59(0.15-2.27)$ \\
\hline Primary education & $0.32(0.09-1.11)$ & 1.00 \\
\hline Secondary or higher education & & $0.57(0.34-0.94)^{*}$ \\
\hline Parent(s) income status & 1.00 & $0.31(0.07-1.40)$ \\
\hline Low & $0.43(0.27-0.69)$ & $0.13(0.03-0.56)$ \\
\hline Moderate & & \\
\hline High & & \\
\hline
\end{tabular}

Type of sanitary material used during the last menstruation

\begin{tabular}{lll} 
Sanitary pad & 1.00 & 1.00 \\
Cloth & $3.68(2.56-5.28)$ & $3.21(2.22-4.63)^{\star}$ \\
Privacy in school & & \\
\hline No & 1.00 & 1.00 \\
\hline yes & $0.68(0.48-0.96)$ & $0.70(0.48-1.02)$ \\
Cultural restrictions & & \\
\hline No & 1.00 & 1.00 \\
\hline Yes & $2.56(1.81-3.68)$ & $2.54(1.76-3.67)^{\star}$
\end{tabular}

*= significance at $\mathrm{P}$-value $<0.05 ; 1=$ Reference category 


\section{Discussion}

Our study investigated the prevalence and predictors of menstruation-related school absenteeism among adolescent girls. The prevalence of school absenteeism was at least one-in-four girls, and the days spent away from school during their last menstruation ranged from one to seven days. Older adolescent girls (18-19 years), use of cloth as a sanitary material in the last menstruation and cultural restrictions were associated with higher odds of school absenteeism, while parent(s) income status (moderate-income) was associated with lower odds of school absenteeism. Mother's education and privacy in school were not associated with school absenteeism at the multivariate regression level.

Our findings of more than a quarter of the adolescent girls missing out school days during their menstrual period highlights the many challenges girls face in managing their menstruation alongside schooling. Missing out school days means missing out critical lessons in class and sometimes examinations, which may never be recovered after they have returned to school. This puts them at a further disadvantage to the boys and has the potential to stall the academic performance of these girls [7, 15]. More so, consistent monthly absenteeism may lead to class repetition and subsequent school dropout of these girls due to poor academic performance [15]. The prevalence of menstruation-related school absenteeism in our study is little lower than the $40 \%$ as found by Mohammed and colleagues [9], in Ghana, $40 \%$ in India by Vashisht and colleagues [7] and 54\% in Ethiopia by Tegegne and Sisay [15]. However, our finding is a little higher when compared to the $20 \%$ menstruation-related school absenteeism as reported in Uganda [8]. The discrepancy could be due to differences in income levels, cultural settings and school infrastructure.

We also found that older adolescent girls were more likely to be absent from school due to menstruationrelated issues when compared with the younger ones. Miiro and colleagues [8], in their study in Uganda reported that missing school during girl's menstrual period was more common among the older adolescents. In a similar study in Indonesia by Davis and colleagues [5], it was also found that girls in higher grades were more likely to report school absenteeism due to menstruation during their last menstrual period. In older adolescents, there is an increased tendency to function independently of their parents and so they tend to make such critical decisions regarding sexual and reproductive health without the involvement of their parents [19]. Older adolescents may therefore shy away from discussing and seeking support from their parents regarding menstrual-related challenges they might be facing in school even if the parents can provide support such as sanitary pad among others. This might be a possible explanation why older adolescent girls are more likely to stay at home during their menstrual period compared to the younger ones. Increased parental income was associated with reduced school absenteeism during menstruation. Girls from parent(s) with moderate-income status are likely to get funds that will cater for their monthly sanitary pads, and this might enhance their school attendance. Our findings aligns with a similar study in Northern Ethiopia [15].

The use of cloth in the last menstruation was associated with higher odds of school absenteeism among the girls. Hennegan and colleagues [6], suggested that the use of unclean cloth as a menstrual absorbent 
was associated with fear of odour, inability to concentrate in school and low confidence in answering questions in class, and this may contribute to abstaining from school during menstruation. In a similar study, Montgomery and colleagues [20], reported that school attendance during menstruation was higher among girls who were supplied with sanitary pads compared to those who were not supplied with sanitary pads. These findings are not surprising because most girls feel the sanitary pad is superior to cloth and therefore they are more comfortable using sanitary pad while in school without the fear of staining themselves and being teased [21]. Similar findings are reported elsewhere in Ghana [10], Ethiopia [15], India [22], and Bhutan [23].

The prevalence of school absenteeism in our study was more common among girls who were culturally restricted during menstruation. The period of adolescence is of particular concern because in most LMICs, cultural restrictions, misconceptions, myths and taboos create barriers which prevents girls from understanding the scientific facts about menstruation. These restrictions limit their daily activities and have the potential to negatively affect their self-esteem, reproductive health and school attendance [24, 25]. For example, in certain cultural settings, menstruating girls are excluded from religious functions, water bodies, certain foods, family homes and from using sanitation facilities meant for public. It is also a taboo for girls to look in the mirror or bath during their menstrual period [24-27]. These girls will not be comfortable to go to school if they have not bathed particularly for a good number of days due to fear that they may be smelly and be teased at school. Studies in certain parts of India have also reported that menstruating girls are prevented from going to school until the period of menstruation is over [26, 27].

Our study had some limitations. The main limitation of the study is that it was a cross-sectional study and we cannot establish causal relationships. Also, our study did not consider some aspects of WASH such as water and soap availability at school. Furthermore, our variables were self-reported and recall bias might have been introduced. However, we expect recall bias to impact less on our outcome variable as it was measured in relation to the last menstrual period.

\section{Conclusion}

More than a quarter of the girls stayed out of school during their menstrual periods. The number of absenteeism during the last menstruation varied from one to seven days. School absenteeism during menstruation was more prevalent among girls who used cloth as a menstrual material in their last menstruation, the older adolescents and those who faced cultural restrictions during their menstrual periods. However, school absenteeism was less common among those whose parent(s) had moderateincome. At policy level, interventions should be aimed at improving the availability of sanitary pads for girls, eliminating cultural restrictions associated with menstruation, and also improving parent(s) income level. We recommend future research on the impact of menstruation-related school absenteeism on the academic performance of school girls.

\section{Abbreviations}


LMIC: Low-and-Middle Income Countries

SHR: Sexual and Reproductive Health

\section{Declarations}

\section{Ethics approval and consent to participate}

The Kwame Nkrumah University of Science and Technology/Komfo Anokye Teaching Hospital Committee on Human Research, Publications and Ethics approved the study (CHRPE/AP/348/16). Administrative permission was also sought from Ghana Education Service as well as heads of the various schools. Written informed consent form was obtained from participants 18 years or older, while same was obtained from parents of participants below 18 years, and those participants assented to the study.

\section{Consent for publication}

Not applicable.

\section{Availability of data and materials}

Data is available from the corresponding author upon request.

\section{Competing interest}

The authors have declared that they have no competing interest.

\section{Funding}

The study was no funded

\section{Authors' contribution}

Conceived and designed the study: MTK, FAZ, JA and EO; conducted interviews: MTK, FAZ and JA; performed data analysis: EO; drafted the manuscript: MTK, EO, FAZ and JA; all authors read and approved the final manuscript.

\section{Acknowledgement}

We acknowledge our participants and all the heads of the various schools.

\section{References}

1. UNICEF. Adolescent demographics. 2019. Available from:

https://data.unicef.org/topic/adolescents/demographics/.[Accessed 20 December 2020] 
2. Plourde KF, Fischer S, Cunningham J, Brady K, McCarraher DR. Improving the paradigm of approaches to adolescent sexual and reproductive health. Reprod Health. 2016;13:72.

3. Santhya KG, Jejeebhoy SJ. Sexual and reproductive health and rights of adolescent girls: Evidence from low- and middle-income countries. Glob Public Health. 2015;10(2):189-221.

4. Woog V, Kågesten A. The Sexual and Reproductive Health Needs of Very Young Adolescents In Developing Countries. Guttmarcher Institute. 2017. Available from: https://www.guttmacher.org/factsheet/srh-needs-very-young-adolescents-in-developing-countries

5. Davis J, Macintyre A, Odagiri M, Suriastini W, Cordova A, Huggett C, et al. Menstrual hygiene management and school absenteeism among adolescent students in Indonesia: evidence from a cross-sectional school-based survey. Trop Med Int Heal. 2018;23(12):1350-63.

6. Hennegan J, Dolan C, Wu M, Scott L, Montgomery P. Measuring the prevalence and impact of poor menstrual hygiene management: A quantitative survey of schoolgirls in rural Uganda. BMJ Open. 2016;6:e012596.

7. Vashisht A, Pathak R, Agarwalla R, Patavegar B, Panda M. School absenteeism during menstruationamongst adolescent girls in Delhi, India. J Family Community Med. 2018;25:163-8.

8. Miiro G, Rutakumwa R, Nakiyingi-Miiro J, Nakuya K, Musoke S, Namakula J, et al. Menstrual health and school absenteeism among adolescent girls in Uganda (MENISCUS): A feasibility study. BMC Womens Health. 2018;18:4.

9. Mohammed S, Larsen-Reindorf RE, Awal I. Menstrual Hygiene Management and School Absenteeism among Adolescents in Ghana: Results from a School-Based Cross-Sectional Study in a Rural Community. Int J Reprod Med. 2020;2020.

10. Montgomery P, Ryus CR, Dolan CS, Dopson S, Scott LM. Sanitary Pad Interventions for Girls ' Education in Ghana: A Pilot Study. PLoS One. 2012;7(10).

11. Grant M, Lloyd C, Mensch B. Menstruation and school absenteeism: Evidence from rural Malawi. Comp Educ Rev. 2013;57(2):260-84.

12. Sommer M, Caruso BA, Sahin M, Calderon T, Cavill S. A Time for Global Action : Addressing Girls ' Menstrual Hygiene Management Needs in Schools. PLOS Med. 2016;13(2):e1001962.

13. Ssewanyana D, Kiiza B, Bitanihirwe Y. Menstrual hygiene management among adolescent girls in sub-Saharan Africa. Glob Health Promot. 2017;0(0):1757-9759.

14. Boosey R, Georgina P, Deave T. Menstrual hygiene management amongst schoolgirls in the Rukungiri district of Uganda and the impact on their education: a cross-sectional study. Pan Afr Med J. 2014;4(3).

15. Tegegne TK, Sisay MM. Menstrual hygiene management and school absenteeism among female adolescent students in Northeast Ethiopia. BMC public heealth. 2014;14.

16. Mohammed S, Emil Larsen-Reindorf R. Menstrual knowledge, sociocultural restrictions, and barriers to menstrual hygiene management in Ghana: Evidence from a multi-method survey among adolescent schoolgirls and schoolboys. PLoS One. 2020;15(10):e0241106. 
17. Acheampong K, Baffour-Awuah D, Ganu D, Appiah S, Pan X, Kaminga A, et al. Prevalence and predictors of dysmenorrhea, its effect, and coping mechanisms among adolescents in Shai Osudoku district, Ghana. Obstet Gynecol Int. 2019;2019.

18. Gumanga SK, Kwame-Aryee RA. Menstrual characteristics in some adolescent girls in accra, ghana. GHANA Med J. 2012;46(1):3-7.

19. World Health Organization. Issues in adolescent health and development. Geneva; 2004. Available from: http://whqlibdoc.who.int/publications/2004/9241591447_eng.pdf.[Accessed 21 December 2020]

20. Montgomery P, Hennegan J, Dolan C, Wu M, Steinfield L, Scott L. Menstruation and the Cycle of Poverty: A Cluster Quasi-Randomised Control Trial of Sanitary Pad and Puberty Education Provision in Uganda. PLoS One. 2016;11(12):e0166122.

21. Hennegan J, Dolan C, Steinfield L, Montgomery P. A qualitative understanding of the effects of reusable sanitary pads and puberty education: Implications for future research and practice. Reprod Health. 2017;14:78.

22. Sivakami M, van Eijk AM, Thakur H, Kakade N, Patil C, Shinde S, et al. Effect of menstruation on girls and their schooling, and facilitators of menstrual hygiene management in schools: Surveys in government schools in three states in India, 2015. J Glob Health. 2019;9(1).

23. UNICEF. Knowledge, Attitude and Practices of Menstrual Hygiene Management by Adolescent schoolgoing girls and nuns in Bhutan [Internet]. 2018. Available from: https://www.unicef.org/bhutan/media/211/file

24. House S, Mahon T, Cavill S. Menstrual hygiene matters; A resource for improving menstrual hygiene around the world. 1st ed. London: WaterAid; 2012. 22-354 p.

25. WaterAid. Is Menstrual Hygiene And Management An Issue For Adolescent Girls? A Comparative Study Of Four Schools In Different Settings Of Nepal. 2009. Available from: http://www.wssinfo.org/fileadmin/user_upload/resources/MENSTRUAL-HYGIENE-MANAGEMENTPaper-for-END-group-1.pdf.[Accessed 16 December 2020]

26. Thakre SB, Thakre SS, Reddy M, Rathi N, Pathak K, Ughade S. Menstrual Hygiene: Knowledge and Practice among Adolescent School Girls of Saoner, Nagpur District. J Clin DIAGNOSTIC Res. 2011;5(5):1027-33.

27. Kumar A, Srivastava K. Cultural and social practices regarding menstruation among adolescent girls. Soc Work Public Health. 2011;26(6):594-604. 